\title{
On the Modern Status of the Universe Rotation Problem
}

\author{
L. M. Chechin \\ V. G. Fessenkov Astrophysical Institute, National Centre for Cosmic Researches and Technologies, \\ National Space Agency, Almaty, Kazakhstan \\ Email: chechin-1m@mail.ru, leonid.chechin@gmail.com
}

Received June 15, 2013; revised July 17, 2013; accepted August 12, 2013

Copyright (C) 2013 L. M. Chechin. This is an open access article distributed under the Creative Commons Attribution License, which permits unrestricted use, distribution, and reproduction in any medium, provided the original work is properly cited.

\begin{abstract}
The brief modern review of the Universe rotation problem is given. Also it was argued the vacuum concept of the Universe rotation, found the galactic coordinates of the Universe rotation axis, based theoretically the concept of the decaleration parameter anisotropy.
\end{abstract}

Keywords: Universe Rotation; Cosmic Vacuum; Anisotropy of Deceleration Parameter

\section{Introduction}

One of the most important global characteristics of the Universe is its rotation. In fact, in article [1] it was emphasized that in contrast to most astronomical objects (such as planets, stars, and galaxies), the Universe as a whole is usually considered to be non-rotational. However, the possibility that the Universe rotates should not be ignored. In fact, the solutions of general relativity corresponding to a rotating Universe have been found in [2-5], directly indicating that a global rotation is physically allowed.

There are many other approaches to constrain the global rotation.

Based on the idea that a global rotation induces a total net spin of galaxies, the global rotation can be limited [6]. Moreover, empirical relations between angular momentum and mass of galaxies/clusters, such as $J \sim M^{\frac{5}{3}}$ for spiral galaxies and $J \sim M^{2}$ for clusters can be explained by the global rotation [7]. The acceleration caused by the global rotation may be used to explain parts of the accelerating expansion of our Universe, and thus the global rotation can be constrained by Supernova type Ia data.

Beside, authors quoted number of previous articles devoted to clarifying this problem and conclude that study of global rotation is of interest in many different aspects of cosmology, and constraint of the rotation speed of the Universe is important.

To develop a model that preserves the homogeneity and isotropy of the mean $\mathrm{CMB}$, they studied the rotation of the Universe as a perturbation in the Robertson-
Walker framework with a cosmological constant. Unlike the Bianchi models, such an approach allows to have non-zero rotation but trivial shear. Since the global rotation does not have any influences on the first-order SachsWolfe effect (SW effect), we need to calculate the metric up to second-order perturbations and find the secondorder SW effect. Then, they constrained the angular speed of the rotation using recent data on CMBA. As the result their model is inhomogeneous with an axial symmetry in general. Moreover, the global rotation in this model is not only radial-dependent, but also time-dependent.

In fact, the angular velocity describes by expression $\Omega(r, \eta)=\Omega_{0} \cdot \frac{r^{2}}{r_{\varepsilon}^{2}} \cdot \frac{a^{3}\left(\eta_{\varepsilon}\right)}{a^{3}(\eta)}$, where $\Omega_{0} \sim 6 \times 10^{-26} \mathrm{~m}^{-1}$ in SI unit. The subscripts " 0 " and " $\varepsilon$ " at radius $r$ and conformal time $\eta$ are denoting the hypersurfaces at origin and last photons scattering in SW effect respectively. In doing this the angular velocity of matter can be negative while the rotation speed of the Universe is always positive and is less than $10^{-9} \mathrm{rad} \cdot \mathrm{y}^{-1}$ at the last scattering surface.

The first experimental evidence of the Universe rotation was done by Birch [8], evidently. In this article he results of a study of the position angles and polarization of high luminosity classical-double radio sources are discussed, taking into account a sample of 94 radio sources. There appears to be strong evidence that the Universe is anisotropic on a large scale, producing position angle offsets in the polarization and brightness distributions of radio sources. These can probably be ex- 
plained on the basis of a rotation of the Universe with an angular velocity of approximately $10^{-13} \mathrm{rad} \cdot \mathrm{y}^{-1}$. Such a rotation would have drastic cosmological consequences, since it would violate Mach's principle and the widely held assumption of large-scale isotropy.

Contrary to this in article [9] it was concluded, from an independent analysis of a new, enhanced sample of radio sources, that there is no evidence for a large-scale anisotropy of this type, or for universal rotation.

Nevertheless, the observational proofing of Universe rotation phenomenon is still important up to the present days. Really, in [10] a preference for spiral galaxies in one sector of the sky to be left-handed or right-handed spirals would indicate a parity violating asymmetry in the overall Universe and a preferred axis was considered. This study uses 15158 spiral galaxies with redshifts $z<0.085$ from the Sloan Digital Sky Survey. An unbinned analysis for a dipole component that made no prior assumptions for the dipole axis gives a dipole asymmetry of $-0.0408 \pm 0.011$ with a probability of occurring by chance of $7.9 \times 10^{-4}$. A similar asymmetry is seen in the Southern Galaxy spin catalog of Iye and Sugai. The axis of the dipole asymmetry lies at approx. $(l, b)=\left(52.0^{\circ}, 68.5^{\circ}\right)$, roughly along that of our Galaxy and close to alignments observed in the WMAP cosmic microwave background distributions. The observed spin correlation extends out to separations $\sim 210 \mathrm{Mpc} / \mathrm{h}$, while spirals with separations $<20 \mathrm{Mpc} / \mathrm{h}$ have smaller spin correlations.

Moreover, in [11] a statistically significant anisotropy of the Hubble diagram at redshifts $z<0.2$ is discovered with the confidence level $>95 \%$. While data from the North Galactic hemisphere favor the accelerated expansion of the Universe, data from the South Galactic hemisphere are not conclusive. The hemispheric asymmetry is maximal toward a direction close to the equatorial poles. The discrepancy between the equatorial North and South hemispheres shows up in the SN calibration. For the $\Lambda \mathrm{CDM}$ model fitted to all available $\mathrm{SNe}$, authors found the same asymmetry. This asymmetry may be the evidence of the Universe rotation axis existence.

It is very important to mark that in article [12] was put forward he question-does the Universe accelerate equally in all directions? Authors employed the union compilation of Type Ia supernovae with a maximum likelihood analysis to search for a dark energy dipole. To approach this problem, authors presented a simple, computationally efficient, and largely model independent method. They opted to weight each SN by its observed error estimate, so poorly measured SNe that deviate substantially from the Hubble law do not produce spurious results. As the result they found, with very low significance, a dipole in the cosmic acceleration directed roughly towards the cosmic microwave background di- pole, but this is almost certainly coincidental. Such dipole indicates the Universe anisotropy and may coincide with the direction of its rotation axis.

Another variant of confirmation the Universe rotation axis existence have been done in article [13].

The novel aspects of the Universe rotation problem were considered in [14]. In this paper author discussed the observational aspects of rotation in the Universe on different scales. He shows dependence between the angular momentum of the structures and their size. The presented observational situation is that the galaxies, their pairs and compact groups have a non-vanishing angular momentum. In the structures of mass corresponding to groups of galaxies, this feature has not been found, while in the clusters and superclusters alignment of galaxy orientation has been actually found. Also it's known that galaxies have net angular momentum due to the fact that we actually measure the rotation curves of galaxies. These facts lead to the conclusion that theories which connect galaxy angular momentum with its surrounding structure are at some extend favored by data. Author shows that in the light of scenarios of galaxy formations this result could be interpreted as an effect of tidal forces mechanism, but it is also consistent with Li's model, in which galaxies form in the rotating Universe.

The article organizes as follows. In second section we argued the vacuum conception of the Universe rotation. In third section we calculate the galactic coordinates of the Universe rotational axis. Forth section devoted to the theoretical basing picked axis in the Universe existence. In conclusion we briefly summarize the results of article.

\section{Vacuum Conception of the Universe Rotation}

But now question arises - if the Universe rotates what is the physical reason of this phenomenon? Such question is very important because in all of previous article the cosmological rotation has been introduced "by hands". One of the first articles was done by Ellis and Olive [15]. They pointed out that an inflationary episode in the very early Universe could solve the rotation problem and thus make the Mach principle redundant as an explanation. The difficulties posed by the rotation-induced shear for the inflationary explanation are avoided if the inflation occurred at the Planck epoch. The problem of the slow rotation of the Universe is addressed by introducing a dimensionless measure of the angular velocity in the early Universe, which must have been less than $5 \times 10^{-28}$ in order to be compatible with present-day upper limits on rotation.

Note that article [16] has been prepared in this trend recently. Its aim is the searching of rotational movement of galaxies, caused by the cosmic vacuum or antigravitational vacuum force and its application to the Universe 
rotation problem.

So, considering a galaxy as the rigid body we'll examine its rotation under acting of vacuum antigravitational force with respect to the center-of-mass point.

In [16] it was argued that elementary vacuum force acted on any volume of a galaxy $d \Omega$ is

$$
\mathrm{d} f_{V}=-\frac{G \mathrm{~d} m_{G}}{R^{2}}=-\frac{G \rho_{G} \mathrm{~d} \Omega}{R^{2}}=2 \frac{G \rho_{V} \mathrm{~d} \Omega}{R^{2}} .
$$

At basing (2.1) it was took into account the vacuum equation of state $p_{V}=-\rho_{V}$ and fact that according to the Friedmann cosmological model the gravitation produces by generalized mass density $\rho_{G}=\rho+3 p$, where $p$ is the substrate pressure. Let $\gamma$ is the angle between vectors $\boldsymbol{r}$ and $\boldsymbol{R}_{0}$. Then under the condition

$r / R_{0} \ll 1$ we find the element of gravitational repelling force

$$
\mathrm{d} f_{V}=\frac{2 G \rho_{V}\left(1+2 \frac{r}{R_{0}} \cos \gamma\right)}{R_{0}{ }^{2}} \mathrm{~d} x \mathrm{~d} y \mathrm{~d} z
$$

and its total expression

$$
f_{V}=\frac{\mathrm{d} U_{V}}{\mathrm{~d} R}=4 \frac{G \rho_{V}}{R_{0}^{3}} \int r \cos \gamma \mathrm{d} x \mathrm{~d} y \mathrm{~d} z,
$$

where integral must be taken over the volume of galaxy and $U_{V}$ is the vacuum potential.

Introducing the vacuum moments $I_{j}$ of the galaxy along axes $\xi, \eta, \varsigma$ and cross-section areas $S_{\eta \zeta}=\iint \mathrm{d} \eta \mathrm{d} \varsigma, S_{\xi \zeta}=\iint \mathrm{d} \xi \mathrm{d} \varsigma, S_{\xi \eta}=\iint \mathrm{d} \xi \mathrm{d} \eta$, corresponding them, it's possible write down the vacuum perturb force and the vacuum potential as follows

$$
\begin{aligned}
& f_{i}=4 \frac{G}{R_{0}^{3}} a_{i k} \cdot I_{j} S_{j} \cdot e_{k}=-\frac{\partial U_{V}}{\partial R_{0}} e_{i} . \\
& U_{V}=-4 G a_{i k} \cdot I_{j} S_{j} \cdot \int \frac{e_{i} e_{j}}{R_{0}^{3}} \mathrm{~d} R_{0},
\end{aligned}
$$

accordingly.

Later on we choose the elliptical galaxy as the model of examining type of galaxies. This lead not only to simplify the process of the analytical solving our problem, but allowed to compare results with the real astronomical database. Consider an elliptical galaxy that have two equal main moments $A=B \neq C$. Than the potential will not depends on angle $\phi$. Hence, it may be equals to zero, i.e. $\phi=0$.

For the chosen shape of galaxy its equations of rotational motion are

$$
\begin{aligned}
& \theta \frac{\mathrm{d} \theta}{\mathrm{d} t}=\tilde{\kappa} \cdot(\theta-2 \psi) \\
& \theta \frac{\mathrm{d} \psi}{\mathrm{d} t}=\tilde{\kappa} \cdot(2 \theta-\psi)
\end{aligned}
$$

where coefficient $\tilde{\kappa}=4 G \cdot I_{j} S_{j} / C \omega R_{0}^{2}$. The system (2.6) is easy transforms to the differential equation of the first order

$$
\frac{\mathrm{d} \theta}{\mathrm{d} \psi}=2\left(1-\frac{1}{2} \frac{\theta}{\psi}\right) /\left(1-2 \frac{\theta}{\psi}\right),
$$

The solution of this equation describes by standard quadrature $\ln \psi=\int \frac{\mathrm{d} u}{F(u)-u}+\hat{C}$, with variable $u=\frac{\theta}{\psi}$, and $\hat{C}=\mathrm{const}[17]$. In [16] it was shown that

$$
\psi(t)=8 \frac{G I_{j} S_{J}}{C \omega R_{0}^{2}} \cdot t .
$$

Basing on this result it is easy to calculate the angular velocity of the elliptical galaxy around $O Z$ axis. In fact, as for this case $\omega=-\dot{\psi}$, than its module equals

$$
\varpi_{V}=\sqrt{8 \frac{G I_{j} S_{j}}{C R_{0}^{2}}}=\text { const . }
$$

This expression describes the angular velocity that galaxy acquires due to the vacuum antigravitation.

Consider, for example, the giant elliptical galaxy NGC 4486 ( $\mathrm{M} 87$ ), that locates from us at distance $R_{0}=15$ MPc, while its proper sizes $l$ equals $60 \mathrm{Kpc}$. With account of above cited vacuum density we estimate the magnitude of Expression (18) now. Putting $I_{j} S_{j} \sim 3 I S \sim \rho_{V} l^{4}$ and assuming that $C \sim l^{2}$, we find $\varpi_{V} \propto \sqrt{8 G \rho_{V}} \cdot \frac{l}{R_{0}} \propto \varpi_{0} \cdot \frac{l}{R_{0}}$. Substitution the needed values into this expression give the following estimation $\varpi_{V}=6.4 \times 10^{-21} \mathrm{sec}^{-1}$.

At the same time analyses of (2.9) shows that its maximal magnitude will be under the condition $l \propto R_{0}$. Then expression for the vacuum angular velocity simplifies and takes on the form

$$
\varpi_{V}=\varpi_{0} \propto \sqrt{G \rho_{V}} .
$$

This expression is possible to interpret as the minimal angular velocity in the Universe that vacuum can create at arbitrary object its numerical value is about $10^{-19} \mathrm{sec}^{-1}$. Hence, the vacuum itself create the identical initial angular velocity at all of cosmic objects. Thus it is possible to say that cosmic vacuum not only creates the microscopic baryonic substance, but pre-determines its global characteristics.

As the condition $l \sim R_{0}$ indicates the passing to the entire Universe it results about its total rotation. As it seems Expression (2.10) is invariant and don't depend nether the frame of reference nor choosing the geometrical sizes of the Universe at given cosmological epoch. Hence, at the earliest stages of Universe evolution, for 
example at baryonic asymmetry stage, when vacuum density was about $10^{-15} \mathrm{~g} / \mathrm{cm}^{3}$ its angular velocity was essentially larger and was equal $\varpi_{V} \sim 10^{-11} \mathrm{sec}^{-1}$. For the very early Universe vacuum density was about $10^{90} \mathrm{~g} / \mathrm{sm}^{3}$ in order and its angular velocity was drastically larger $\varpi_{V} \sim 10^{42} \mathrm{sec}^{-1}$.

Mark, at such angular velocity the border of Universe moved with linear velocity not larger the speed of light. In fact, as the sizes of Universe at born time were Planckian type, i.e. $\ell_{P l} \approx 1.6 \times 10^{-35} \mathrm{~m}$, than linear velocity of the Universe border will be

$v=\ell_{P l} \cdot \varpi_{V} \sim 10^{7} \mathrm{~m} \cdot \mathrm{sec}^{-1}$. This velocity is smaller that speed of light truly, that is must be in realty.

What about the observable angular velocity of the Universe at the present epoch? In papers [6,7] there were given following estimations of its angular velocity $\varpi_{0} \sim 10^{-21} \mathrm{sec}^{-1}$. In article [1], that was done on the base of novel experimental data on the relic radiation distribution divergence from Gaussian, was shown that the Universe angular velocity must be smaller than $\varpi_{0} \sim 10^{-17} \mathrm{sec}^{-1}$.

From these estimations it result that Universe angular velocity must be in the following range

$10^{-21} \mathrm{sec}^{-1} \leq \varpi_{0} \leq 10^{-17} \mathrm{sec}^{-1}$. Our previous estimate of the Universe rotation $10^{-19} \mathrm{sec}^{-1}$, as it seems, is in good correlation with the specified interval.

At the same time the peculiarity of quoted articles is the Universe rotation searching in the framework of relativistic cosmology and its determination by a baryonic substrate. In contrary, in our case the Universe rotation determines by Newtonian's mechanics and is the consequence of vacuum presence. That is why the ratio of Universe angular velocity to the Hubble parameter is about unit, i.e. $\left(\frac{\varpi}{H}\right)_{0} \sim 1$. From this follows the correctness of Gamov's remark [18] that the unique reason for Universe expansion and its rotation must be. If take into account that vacuum creates all baryonic substance, it is the moving force of the total Universe evolution, hence.

\section{Galactic Coordinates of the Universe Rotational Axis}

Talking about the Universe rotation it is not ignore the difficulty connected with the determination of the Universe rotational axis and its orientation in the space. Such question was considered by number authors, but from observational point of view preferentially. We'll examine it from the theoretical positions basing on the coordinate transformation that expressed by Euler angles and galactic coordinates.

Remind that galactic coordinates system this is the system with origin placed on the Sun and extended through the center of Galaxy. Its plane coincides with the plane of Galaxy dick. In doing this, its latitude $b$ is measured from galaxies plane to an object and takes magnitudes from $-90^{\circ}$ up to $+90^{\circ}$. Galactic longitude $l$ is measured at the Galaxy plane from the baseline connected the Sun and galactic center up to the baseline connected the Sun and object. Counting directs to the same way that the right ascension in the second equatorial coordinates. Therefore the galactic longitude puts in limits from $0^{\circ}$ up to $360^{\circ}$.

The position of object in galactic coordinates describes by matrix expression

$$
\boldsymbol{r}_{g}=\left(\begin{array}{c}
\cos b \cos l \\
\cos b \sin l \\
\sin l
\end{array}\right)\left(\begin{array}{lll}
\boldsymbol{i}_{g} & \boldsymbol{j}_{g} & \boldsymbol{k}_{g}
\end{array}\right)
$$

where $\left(\begin{array}{c}\cos b \cos l \\ \cos b \sin l \\ \sin l\end{array}\right)$ is the matrix of galactic coordinates while $\left(\begin{array}{lll}\boldsymbol{i}_{g} & \boldsymbol{j}_{g} & \boldsymbol{k}_{g}\end{array}\right)$-its basis vectors.

The radius-vector $\boldsymbol{r}$ of a celestial object that is expressed by equatorial coordinates $(\alpha, \delta)$ may be decomposed by basis vectors of other coordinates of galactic coordinates $(l, b)$, in particular. As equatorial coordinates $(\alpha, \delta)$ is connected with the Euler coordinates $(\theta, \psi, \varphi)$ taken under the condition $(\varphi=0)$ by the equalities $\delta=\theta$ and $\alpha=\psi-2 \pi$, we have the following relationship

$$
\begin{aligned}
\boldsymbol{r} & =\left(\begin{array}{lll}
\boldsymbol{i} & \boldsymbol{j} & \boldsymbol{k}
\end{array}\right)\left(\begin{array}{c}
\cos \theta \cos \psi \\
\cos \theta \sin \psi \\
\sin \psi
\end{array}\right) \\
& =\left(\begin{array}{lll}
\boldsymbol{i}_{g} & \boldsymbol{j}_{g} & \boldsymbol{k}_{g}
\end{array}\right)\left(\begin{array}{c}
\cos b \cos l \\
\cos b \sin l \\
\sin l
\end{array}\right)
\end{aligned}
$$

To find the explicit form of transformation between two coordinate systems it is necessary to calculate the rotation matrix from first basis unit vectors to second one. For example, as it follows from above the matrix of galactic coordinates is

$$
\begin{aligned}
\left(\begin{array}{c}
\cos b \cos l \\
\cos b \sin l \\
\sin l
\end{array}\right) & =\left(\begin{array}{l}
\mathbf{i}_{g} \\
\boldsymbol{j}_{g} \\
\boldsymbol{k}_{g}
\end{array}\right)\left(\begin{array}{lll}
\boldsymbol{i} & \boldsymbol{j} & \boldsymbol{k}
\end{array}\right)\left(\begin{array}{c}
\cos \theta \cos \psi \\
\cos \theta \sin \psi \\
\sin \psi
\end{array}\right) \\
& =A_{g}\left(\begin{array}{c}
\cos \theta \cos \psi \\
\cos \theta \sin \psi \\
\sin \psi
\end{array}\right)
\end{aligned}
$$

Also remind that ort $\boldsymbol{i}$ is directed to the point of spring equinox; ort $\boldsymbol{j}$ - to the point of right ascension 
that equals $\delta=\frac{\pi}{2}$; ort $\boldsymbol{k}$-to the North celestial pole. Finally, we have to calculate the matrix

$A_{g}=\left(\begin{array}{l}\boldsymbol{i}_{g} \\ \boldsymbol{j}_{g} \\ \boldsymbol{k}_{g}\end{array}\right)\left(\begin{array}{lll}\boldsymbol{i} & \boldsymbol{j} & \boldsymbol{k}\end{array}\right)$. Basing on the magnitudes of angles for right ascension and declination, defined for the B1950 epoch ${ }^{1}$, it possible to show that [19]

$$
A_{g}=\left(\begin{array}{ccc}
-0.05 & -0.87 & -0.48 \\
0.49 & -0.44 & 0.75 \\
-0.87 & -0.20 & 0.45
\end{array}\right) \text {. }
$$

For next calculations find the concrete magnitudes of Euler angles whose general expression was found in Section 2 .

From its results we get

$$
\psi \sim \theta \approx 8 \pi G \rho_{V} \cdot \frac{T}{\omega},
$$

where $T$ is the timelife of our Universe. Setting $T=13.7 \times 10^{9} \mathrm{y} \approx 4.3 \times 10^{17} \mathrm{sec}$, angular velocity $\omega=2.7 \times 10^{-18} \mathrm{sec}^{-1}$ and having in mind that $G=6.7 \times 10^{-8} \mathrm{~cm}^{3} \cdot \mathrm{g}^{-1} \cdot \mathrm{sec}^{-2}, \quad \rho_{V} \approx 4.2 \times 10^{-30} \mathrm{~g} \cdot \mathrm{cm}^{-3}$, $1 \mathrm{rad}=57.3^{\circ}$, we get following estimations

$$
\psi \approx \theta \approx 63.1^{\circ} \text {. }
$$

As $\sin \left(63.1^{\circ}\right) \approx 0.89$ and $\cos \left(63.1^{\circ}\right) \approx 0.45$ the explicit form of the galactic coordinates matrix will be

$$
\begin{aligned}
& \left(\begin{array}{c}
\cos b \cos l \\
\cos b \sin l \\
\sin b
\end{array}\right) \\
& =\left(\begin{array}{ccc}
-0.05 & -0.87 & -0.48 \\
0.49 & -0.44 & 0.75 \\
-0.87 & -0.20 & 0.45
\end{array}\right)\left(\begin{array}{c}
0.45 \cdot 0.45 \\
0.45 \cdot 0.89 \\
0.89
\end{array}\right) \\
& =\left(\begin{array}{ccc}
-0.05 & -0.87 & -0.48 \\
0.49 & -0.44 & 0.75 \\
-0.87 & -0.20 & 0.45
\end{array}\right)\left(\begin{array}{c}
0.20 \\
0.40 \\
0.89
\end{array}\right)=\left(\begin{array}{c}
-0.78 \\
0.51 \\
0.15
\end{array}\right)
\end{aligned}
$$

From this we get the system of three equations for two variables $b$ and $l$

$$
\left.\begin{array}{l}
\cos b \cos l=-0.78 \\
\cos b \sin l=0.51 \\
\sin b=0.15
\end{array}\right\} .
$$

From two last of them we find $l \approx 44^{\circ}, b \approx 9.0^{\circ}$. Compare these magnitudes with results of article [20,21],

\footnotetext{
${ }^{1}$ Passage to coordinates defined for the current epoch J2000.0 don't
} change our subsequent result. where the galactic coordinates of the Universe rotation axis were determined as $(l, b)=\left(314_{+20^{\circ}}^{\circ-13^{\circ}} ; 28_{+11^{\circ}-33^{\circ}}\right)$. It's easy to see that the axis passes through the Sun and point $O$ with angular coordinates $(l, b) \approx\left(44.0^{\circ} ; 9.0^{\circ}\right)$ will coincide with the axis passes through the Sun and point with angular coordinates $(l, b) \approx\left(224 \cdot 0^{\circ} ; 9 \cdot 0^{\circ}\right)$. That is why it possible concludes that Universe rotation axis has this coordinates namely ${ }^{2}$.

The divergence between observational angular coordinates $(l, b)=\left(314_{+20^{\circ}}^{\circ-13^{\circ}} ; 28_{+11^{\circ}}^{\circ-33^{\circ}}\right)$ and our theoretically predicted coordinates $(l, b) \approx\left(224.0^{\circ} ; 9.0^{\circ}\right)$ explains by accounting the vacuum density only at the calculation of Euler coordinates. Hence, except vacuum density it is necessary take into account densities of dark matter and baryonic matter that are the integral components of any galaxies structure. Nevertheless, our approach demonstrates the possible way of theoretical deducing the space orientation of the Universe rotational axis.

Its fruitfulness seems through the prism of Universe evolution, already. In fact, as the magnitude of vacuum density is different at different epochs of its evolution, the space orientation of the rotational axis changes, also. For instance, at the baryonic asymmetry epoch when vacuum density $\rho_{V} \sim 10^{-15} \mathrm{~g} / \mathrm{cm}^{3}$, angular velocity $\varpi_{V} \sim 10^{-11} \mathrm{sec}^{-1}$, timelife $T \sim 10^{-10} \mathrm{sec}$, than Euler angle $\psi \sim\left(10^{-18}\right)^{\circ} \rightarrow 0^{\circ}$. So, the angular coordinates of the rotational axis where $(l, b) \approx\left(90^{\circ} ; 0^{\circ}\right)$.

\section{Basing the Picked Axis in the Universe}

Now we mention the fundamental article [11], once again, where the asymmetry of Hubble's diagrams for the North and the South sky hemispheres was searched. This asymmetry cannot be explains by peculiar motion of the observer, but most apparently due to the any bulk flow along the direction $\left((l, b)=\left(300^{\circ} ; 0^{\circ}\right)\right)$ in the Universe existence that earlier was argued in article [22]. Recently R.-G. Cai and Z.-L. Tuo [21] determined more precisely this direction $(l, b)=\left(314_{+20^{\circ}}^{\circ-13^{\circ}}, 28_{+11^{\circ}}^{\circ-33^{\circ}}\right)$ and found the maximum anisotropy of the deceleration parameter

$$
\frac{\Delta q}{q}=0.76_{+0.41}^{-0.46} \text {. }
$$

Evidently, these results are possible to summaries as follows, our Universe is anisotropic in realty and possesses by any principal space axis. That is why the cosmological deceleration parameter will be anisotropic, also and must be depend on the principal space direction

${ }^{2}$ Usage of the first equation in (3.8) leads to the approximately same result. 
in definite way. These statements require theoretical basing the direction dependence of the cosmological deceleration parameter phenomenon.

Our searching we start from the well-known results. For the uniform isotropic metric of the space-flat Universe $(k=0)$ the Einstein's equations for the scale factor $a(t)$ are [23]

$$
\begin{aligned}
& \ddot{a}=-\frac{4 \pi G}{3}(\rho+3 p) a, \\
& \left(\frac{\dot{a}}{a}\right)^{2}=-\frac{8 \pi G}{3} \rho, \\
& \dot{\rho} a+3(\rho+p) \dot{a}=0 .
\end{aligned}
$$

Put that scale factor expresses as $a=a_{0}+\delta a$, where $a_{0}$ is the distance in uniform space, while $\delta a-$ small addition (perturb term) for describing the possible space anisotropy. Putting it into the Newtonian Equation (4.1) we get two equations - basic one

$$
\frac{\mathrm{d}^{2} a_{0}}{\mathrm{~d} t^{2}}=\frac{\mathrm{d} v_{0}}{\mathrm{~d} t}=-\frac{4 \pi G}{3} \rho a_{0}
$$

and perturb one

$$
\frac{\mathrm{d}^{2} \delta r}{\mathrm{~d} t^{2}}=-\frac{4 \pi G}{3} \rho \delta r .
$$

Later on these equations will be considered independently each other.

Performing the above mentioned Tolman transformation $\rho \rightarrow \rho+3 p$ [23] and substituting it into (4.1) for the case of vacuum $\left(\rho=\rho_{v}, \quad p=-\rho_{v}\right)$ we find equation that get the inflationary regime of the Universe expanding

$$
a_{0}=R \cdot \exp \left(\sqrt{\frac{8 \pi G}{3} \rho_{v}} \cdot t\right)=R \cdot \exp \left(H_{0} \cdot t\right) .
$$

It leads to the Hubble expansion law

$$
v_{0}=\frac{\mathrm{d} a_{0}}{\mathrm{~d} t}=\dot{a}_{0}=\sqrt{\frac{8 \pi G}{3} \rho_{v}} \cdot a_{0}=H_{0} a_{0}
$$

and to the corresponding acceleration

$$
\ddot{a}_{0}=H_{0}^{2} a_{0} .
$$

Now consider the Equation (4.5). Suppose that in this equation $\rho=\rho_{b}$, where $\rho_{b}$ is the baryonic substance density. The baryonic substance pressure $p_{b}$ let equals zero, for simplicity.

Last requirement means considering the presence of two-component substance - cosmic vacuum and baryonic dust - in the Universe, that are not interact each other in the basic approximation.
By introducing the designation $\Omega^{2}=\frac{4 \pi G}{3} \rho_{b}$, from (4.5) it follows

$$
\frac{\mathrm{d}^{2} \delta a}{\mathrm{~d} t^{2}}+\Omega^{2} \delta a=0
$$

This oscillatory-type equation possesses by two roots

$$
\delta a_{ \pm}= \pm \delta R \cdot \exp (i \Omega \cdot t)= \pm \delta R \cdot \cos (\Omega \cdot t) .
$$

They lead to the presence of two perturb (with respect to (4.7)) velocities

$$
\begin{aligned}
\delta v_{+} & =\frac{\mathrm{d} \delta a_{+}}{\mathrm{d} t}=+\delta R \cdot i \Omega \exp (i \Omega \cdot t) \\
& =-\delta R \cdot \Omega \cdot \sin \Omega t \\
\delta v_{-} & =\frac{\mathrm{d} \delta a_{-}}{\mathrm{d} t}=-\delta R \cdot i \Omega \exp (i \Omega \cdot t) \\
& =+\delta R \cdot \Omega \cdot \sin \Omega t
\end{aligned}
$$

and two corresponding accelerations

$$
\begin{aligned}
& \frac{\mathrm{d}^{2} \delta a_{+}}{\mathrm{d} t^{2}}=\frac{\mathrm{d} \delta v_{+}}{\mathrm{d} t}=-\Omega^{2} \cdot \delta a_{+} \\
& \frac{\mathrm{d}^{2} \delta a_{-}}{\mathrm{d} t^{2}}=\frac{\mathrm{d} \delta v_{-}}{\mathrm{d} t}=-\Omega^{2} \cdot \delta a_{-}
\end{aligned} .
$$

From physical viewpoint expressions (4.10)-(4.12) mean that presence of baryonic dust matter creates two space-opposite fluxes that are propagate on the background of expanding and accelerating "Hubble vacuum flux" along the Universe rotation axis. That is why it possible writes down the expressions for total distance, velocity and acceleration of any probe particle (galaxy)

$$
\begin{aligned}
& a=a_{0}\left(1 \pm \frac{\delta a}{a_{0}}\right) ; \dot{a}=\dot{a}_{0}\left(1+\frac{\delta \dot{a}}{\dot{a}_{0}}\right) ; \\
& \ddot{a}=\ddot{a}_{0}\left(1+\frac{\delta \ddot{a}}{\ddot{a}_{0}}\right)
\end{aligned}
$$

Thus the cosmological deceleration parameter $q$ with the accuracy no higher than $\frac{\delta r}{r_{0}} \sim \frac{\delta \dot{r}}{\dot{r}_{0}} \sim \frac{\delta \ddot{r}}{\ddot{r}_{0}}<1$ is

$$
\begin{aligned}
q_{ \pm} \approx & -1 \pm 3 \frac{\delta R \cdot \cos \Omega t}{r_{0}} \mp 2 \frac{\delta R \cdot \Omega \cdot \sin \Omega t}{H_{0} r_{0}} \\
& \pm \frac{\delta R \cdot \Omega^{2} \cdot \cos \Omega t}{H_{0}{ }^{2} r_{0}}
\end{aligned} .
$$

Basing on the definitions of $H_{0}$ and $\Omega$ we introduce the new coefficient $\kappa=\frac{\Omega}{H_{0}}=\sqrt{\frac{\rho_{b}}{2 \rho_{v}}}$. As in unit of the critical density $\rho_{b} \sim 0.04$ and vacuum density 
$\rho_{v} \sim 0.7$, coefficient $\kappa \approx 0.17$, henceforth.

From (4.14) it is possible find the relative acceleration difference between two baryonic fluxes with respect to the "Hubble vacuum flux",

$$
\frac{\Delta q}{q_{0}}=2 \frac{\delta R}{R}\left[(3+\kappa) \frac{\cos \kappa H_{0} t}{\exp H_{0} t}-2 \kappa \frac{\sin \kappa H_{0} t}{\exp H_{0} t}\right] .
$$

Assuming that for modern epoch $H_{0} t \sim 1$ we approximately get $\frac{3+\kappa}{\mathrm{e}^{1}} \sim 1.2$. Hence, the first term in right side of (4.15) tends to 1.2, while the second term tends to zero. So,

$$
\frac{\Delta q}{q_{0}} \approx 2.4 \frac{\delta R}{R}
$$

Basing on our assumption $\frac{\delta r}{r_{0}}<1$, that was argued earlier, we may put that it will satisfy if the ratio

$\left(\frac{\delta R}{R}\right)_{\max } \leq 0.2$. This leads to the estimation

$\left(\frac{\Delta q}{q_{0}}\right)_{\max } \leq 0.48$ that is in good correlation (case of the upper magnitude index) with the value

$\left(\frac{\Delta q}{q_{0}}\right)_{\max }=0.76_{+0.41}^{-0.46}$ in [22].

\section{Conclusions}

So, we briefly reviewed the modern status of the Universe rotation problem. In numbers of examined articles, this problem was considered from different viewpoints and done the following common result-our Universe rotate, most probably. On the this statement grounding it was considered the vacuum concept of the Universe rotation, found the galactic coordinates of the Universe axis rotation and based the concept of the deceleration parameter anisotropy along this axis.

It also was shown that all of our theoretical results have the real observational confirmation.

\section{Acknowledgements}

I would like to express my gratitude to Professor G. F. Smoot III for discussion the Universe rotation problem during his visit the VI Astana world economical forum, 22-24 May 2013, Kazakhstan.

\section{REFERENCES}

[1] S. C. Su and M. C. Chu, The Astrophysical Journal, Vol.
703, 2009, pp. 354-361. doi:10.1088/0004-637X/703/1/354

[2] K. Gödel, Reviews of Modern Physics, Vol. 21, 1949, pp. 447-450. doi:10.1103/RevModPhys.21.447

[3] S. Hawking, MNRAS, Vol. 142, 1969, pp. 129-141.

[4] J. D. Barrow, R. Juszkiewicz and D. N. Sonoda, MNRAS, Vol. 213, 1985, pp. 917-943.

[5] J. D. Barrow and C. G. Tsagas, Classical and Quantum Gravity, Vol. 21, 2004, pp. 1773-1790. doi:10.1088/0264-9381/21/7/005

[6] L. X. Li, General Relativity and Gravitation, Vol. 30, 1998, pp. 497-507. doi:10.1023/A:1018867011142

[7] W. Godlowski and M. Szydlowski, General Relativity and Gravitation, Vol. 35, 2003, pp. 2171-2187. doi:10.1023/A:1027301723533

[8] P. Birch, Nature, Vol. 298, 1982, pp. 451-454. doi:10.1038/298451a0

[9] M. F. Bietenholtz and P. P. Kronberg, The Astrophysical Journal, Part 2, Vol. 287, 1984, pp. L1-L2. doi: $10.1086 / 184383$

[10] M. J. Longo, Physics Letters B, Vol. 699, 2011, pp. 224-229.

[11] D. J. Schwarz and B. Weinhorst, Astronomy \& Astrophysics, Vol. 474, 2007, pp. 717-729. doi:10.1051/0004-6361:20077998

[12] R. Cooke and D. Lynden-Bell, MNRAS, Vol. 401, 2010, pp. 1409-1414. doi:10.1111/j.1365-2966.2009.15755.x

[13] L. M. Chechin, International Journal of Astronomy and Astrophysics, Vol. 2, 2012, pp. 195-198. doi:10.4236/ijaa.2012.24024

[14] W. Godlowski, IJMPD, Vol. 20, 2011, pp. 1643-1674. doi:10.1142/S0218271811019475

[15] J. Ellis and K. A. Olive, Nature, Vol. 303, 1983, pp. 679681. doi:10.1038/303679a0

[16] L. M. Chechin, Astron. Rep., Vol. 87, 2010, pp. 784-788. doi:10.1134/S1063772910080044

[17] V. V. Stepanov, "Course of the Differential Equations," GIFML, Moscow, 1958.

[18] G. Gamov, Nature, Vol. 158, 1946, p. 549. doi:10.1038/158549a0

[19] V. Ye. Zharov, "The Spherical Astronomy," Vek 2, Moscow, 2006.

[20] M. J. Hudson, R. J. Smith, J. R. Lucey and E. Branchini, $M N R A S$, Vol. 352, 2004, pp. 61-75. doi:10.1111/j.1365-2966.2004.07893.x

[21] R.-G. Cai and Z.-L. Tuo, arXiv:1109.0941v4 [astro-ph. CO], 2011.

[22] Y. Itoh, K. Yahata and M. Takada, arXiv:astro-ph/0912. 1460v2.

[23] Ya. B. Zel'dovich and I. D. Novikov, "Relativistic Astrophysics, 2. The Structure and Evolution of the Universe," University of Chicago Press, Chicago, 1983. 